\title{
Mosaic 22q13 deletions: evidence for concurrent mosaic segmental isodisomy and gene conversion
}

\author{
Maria Clara Bonaglia*,1,8, Roberto Giorda ${ }^{1,8}$, Silvana Beri ${ }^{1}$, Stefania Bigoni ${ }^{2}$, Alberto Sensi ${ }^{2}$, \\ Anna Baroncini ${ }^{3}$, Antonella Capucci ${ }^{3}$, Cristina De Agostini ${ }^{1}$, Rhian Gwilliam ${ }^{4}$, \\ Panos Deloukas ${ }^{4}$, Ian Dunham ${ }^{4,5}$ and Orsetta Zuffardi ${ }^{6,7}$
}

\begin{abstract}
${ }^{1}$ Eugenio Medea Scientific Institute, Bosisio Parini, Lecco, Italy; ${ }^{2}$ Department of Experimental and Diagnostic Medicine, Medical Genetic Section, University of Ferrara, Ferrara, Italy; ${ }^{3}$ UO Genetica Medica, AUSL Imola, Imola, Italy; ${ }^{4}$ The Wellcome Trust Sanger Institute, Hinxton, Cambridge, UK; ${ }^{5}$ European Bioinformatics Institute (EBI) Wellcome Trust Genome Campus, Hinxton, Cambridge, UK; ${ }^{6}$ Biologia Generale e Genetica Medica, Università di Pavia, Pavia, Italy; ${ }^{7}$ Fondazione IRCCS C Mondino, Pavia, Italy
\end{abstract}

Although $22 q$ terminal deletions are well documented, very few patients with mosaicism have been reported. We describe two new cases with mosaic 22q13.2-qter deletion, detected by karyotype analysis, showing the neurological phenotype of 22q13.3 deletion syndrome. Case 1 represents an exceptional case of mosaicism for maternal 22q13.2-qter deletion (45\% of cells) and 22q13.2-qter paternal segmental isodisomy ( $55 \%$ of cells). This complex situation was suspected because cytogenetic, FISH and array-CGH analyses showed the presence of an $8.8 \mathrm{Mb}$ mosaic $22 q 13.2$-qter deletion, whereas microsatellite marker analysis was consistent with maternal deletion without any evidence of mosaic deletion. Molecular analysis led to the definition of very close, but not coincident, deletion and uniparental disomy (UPD) break points. Furthermore, we demonstrated that the segmental UPD arose by gene conversion in the same region. In Case 2, mosaicism for a paternal $8.9 \mathrm{Mb} 22$ 13.2-qter deletion (73\% of cells) was detected. In both patients, the level of mosaicism was also verified in saliva samples. We propose possible causative mechanisms for both rearrangements. Although the size of the deletions was quite similar, the phenotype was more severe in Case 2 than in Case 1. As maternal UPD 22 has not been generally associated with any defects and as the size of the deletion is very similar in the two cases, phenotype severity is likely to depend entirely on the degree of mosaicism in each individual.

European Journal of Human Genetics (2009) 17, 426-433; doi:10.1038/ejhg.2008.195; published online 15 October 2008

Keywords: 22q13 deletion; mosaicism; uniparental disomy

\section{Introduction}

To date, thanks to the increasing use of subtelomeric FISH tests, more than 100 cases with monosomy 22q13.3 have been reported. Among these, very few patients showed

*Correspondence: Dr MC Bonaglia, IRCCS Eugenio Medea, Via Don Luigi Monza, 20, 23842 Bosisio Parini, Lecco, Italy.

Tel: + 39031877 111; Fax: + 39031877 499;

E-mail: clara.bonaglia@bp.Inf.it

${ }^{8}$ These authors contributed equally to this study.

Received 22 July 2008; revised 11 September 2008; accepted 12 September 2008; published online 15 October 2008 mosaicism for the 22q13.3 deletion; in fact, the majority of mosaic 22q13 deletions is the product of ring chromosome 22 or of a more complex structural chromosome 22 aberration involving a chromosome deletion and an inverted duplication combined with ring chromosome formation. ${ }^{1-6}$

We report two new cases of mosaicism for the $\operatorname{del}(22)(\mathrm{q} 13.2)$ diagnosed by routine conventional highresolution G-banded karyotype and refined by array CGH analysis. In one case, molecular characterization revealed additional mosaic segmental uniparental disomy (UPD) in 
the 'normal' cell line. Both patients showed all the main features of 22q13.3 deletion syndrome, suggesting that SHANK3 gene haploinsufficiency ${ }^{7-10}$ is sufficient to express the neurological phenotype of the syndrome even in mosaic.

\section{Methods}

Two patients with mosaic 22q13 deletion were ascertained through routine karyotype analysis. Clinical details are provided as Supplementary files.

FISH experiments were performed with the ARSA probe (Vysis) and 22q-cosmid clones n66c4, n85a3, n94h12, and n1g3 as previously reported. ${ }^{11}$ Array-based CGH was performed using the Agilent Human Genome CGH Microarray Kit $44 \mathrm{~B}$ as previously described. ${ }^{12}$

Genomic DNA was extracted from probands' blood, EBV cell lines, parents' blood, and saliva with standard protocols. Somatic cell hybrid clones were generated as in Giorda et al. ${ }^{13}$

The UCSC Genome Browser (May 2004 assembly; http://genome.ucsc.edu/) maps and sequence were used as references. Genotyping of polymorphic sequence-tagged sites was performed by amplification with primers labeled with fluorescent probes followed by analysis on an ABI 310 Genetic Analyzer (Applied Biosystems). Amplifications were performed with AmpliTaq Gold (Applied Biosystems) using standard protocols.

Further genotyping was performed using the Illumina HumanHap500 single beadchip with $750 \mathrm{ng}$ of genomic DNA at a standard concentration of $50 \mathrm{ng} / \mathrm{ml}$. The process conformed to the manufacturer's protocols. The Illumina Beadstudio algorithm was used to call genotypes with manual analysis to confirm cluster boundaries.

Long-range PCRs were performed with JumpStart Red ACCUTaq LA DNA polymerase (Sigma). Fragments were cloned in pCR-4 TOPO using a TOPO TA cloning kit (Invitrogen). All sequencing reactions were performed with a Big Dye Terminator Cycle Sequencing kit (Applied Biosystems) and run on an ABI Prism 3130xl Genetic Analyzer.

For Case 1 break point cloning, two chromosome 22specific primers adjacent to the deletion break points were designed and used for nested ACP-PCR. ${ }^{11}$ PCR products were both directly sequenced and cloned, followed by sequencing of individual clones.

Dilution cloning was performed by seeding 96-well plates with cells from Case $1 \mathrm{EBV}$ line diluted to 0.3 cells per well in RPMI medium containing 10\% FCS. DNA was extracted from the resulting clones with DNAzol.

\section{Results}

\section{Clinical characteristics}

Clinical features are summarized in Table 1 . As in nonmosaic 22q13 deletion, both patients showed all the main features of 22q13.3 deletion syndrome (global developmental delay, delayed speech, accelerated growth, neonatal hypotonia, minor dysmorphism, and autistic-like behaviour). The psychomotor delay was more severe in Case 2 than in Case 1.

Cytogenetic analysis revealed in both cases a de novo mosaic 22q13.2-qter deletion.

The karyotype was as follows:

Case 1: 46,XX,del(22)(pter $\rightarrow q 13.2::)$ [45]/46,XX [55].

Case 2: 46,XX,del(22)(pter $\rightarrow q 13.2::)$ [73]/46,XX [27].

FISH analysis with ARSA and $22 \mathrm{q}$ cosmid probes confirmed a pure terminal 22q13 deletion in both cases. In particular, in Case 1 we also found one metaphase, out of 200 examined, containing three non-deleted chromosomes 22.

Array CGH analysis demonstrated that the deletion encompasses the terminal $8.8 \mathrm{Mb}$ (Figure 1a) and $8.9 \mathrm{Mb}$ (Figure 1b) in Case 1 and in Case 2, respectively.

Typing of polymorphic chromosome 22 markers in the deleted interval in the probands and their parents demonstrated the following:

Case 1: the 22q13 deletion was of maternal origin without any evidence of mosaic deletion (Figure 1c and Table 2).

Case 2: the 22q13 deletion involved the paternal chromosome 22, with evidence of deletion mosaicism (Figure 1d and Supplementary Table 1).

\section{Somatic cell hybrids, break point cloning, and sequencing}

As in Case 1 cytogenetic investigations had clearly demonstrated a mosaic 22q13.2-qter deletion, whereas microsatellite analysis showed a maternal deletion without any evidence of mosaicism, the presence of segmental paternal isodisomy in the 'normal' cell line was supposed. We tested this hypothesis on somatic cell hybrid clones made by fusing Case 1's lymphoblastoid line with a Hamster cell line.

We isolated and analyzed 76 clones with informative chromosome 22 markers. We found three clones with a normal paternal chromosome 22, one with a deleted maternal 22, one with a non-deleted maternal chromosome 22 with segmental paternal UPD of its distal portion (Table 2).

We mapped and cloned with ACP-PCR the break point of the deleted chromosome 22. The deletion break point is followed by a TT dinucleotide and a series of (AGGGTT) telomeric repeats, and it localizes approximately in the middle of a $1.3 \mathrm{~kb}(\text { GAAA })_{n}(\text { GAAAAA })_{n}$ simple repeat in intron 5 of the CENPM gene (Figure 2a, Supplementary Figure 1). The repeat was very unstable when cloned in bacteria and generated clones containing a variety of deletions. This probably explains why the current Genome Map shows it as a $131 \mathrm{bp}$ sequence (chr22:4066572640665857). 
Table 1 Summary of the clinical features of mosaic $22 q 13$ deletion cases

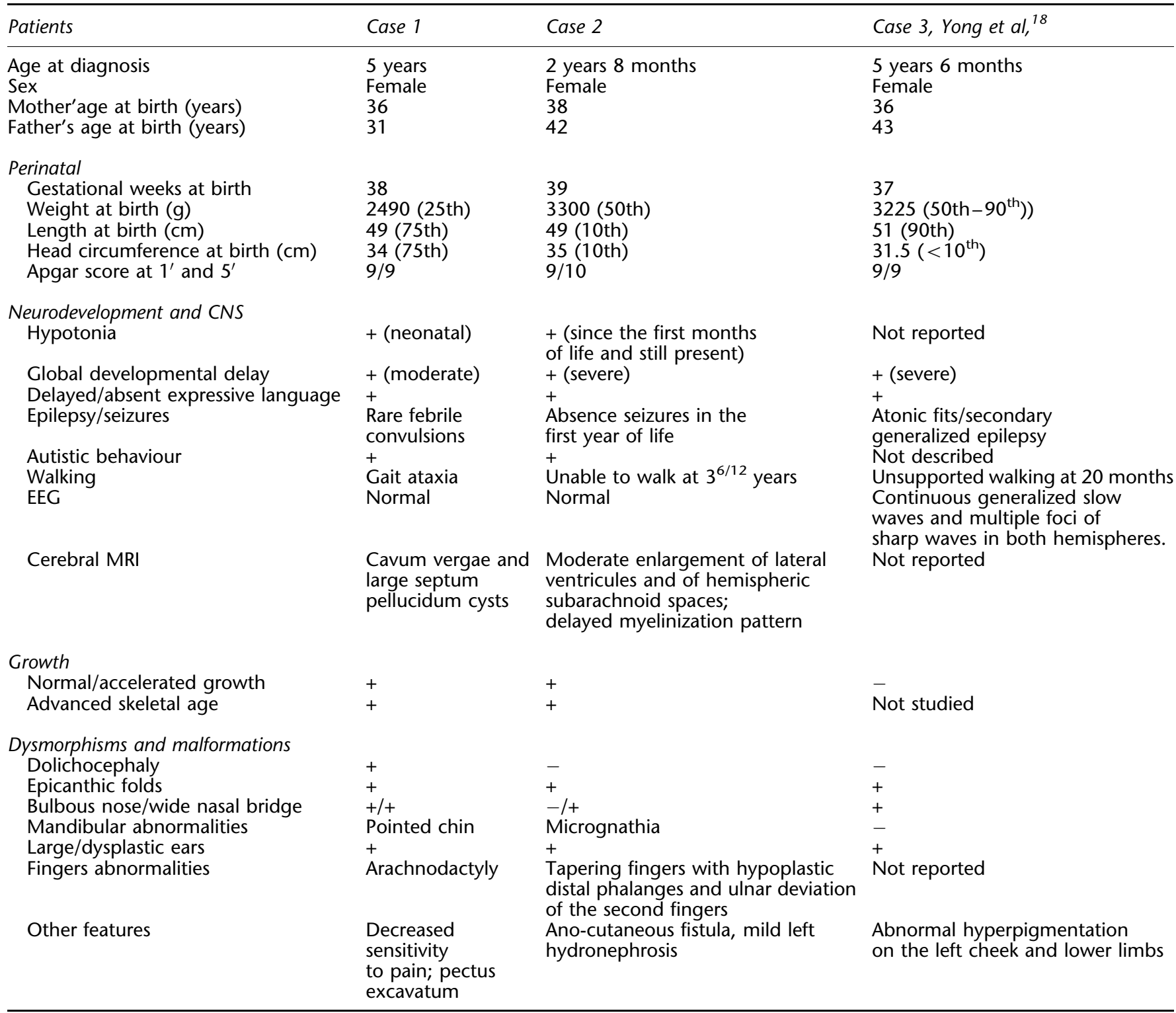

The proband's non-deleted maternal chromosome 22 carries the maternal allele at four polymorphisms (1226-1246) located downstream of the deletion break point (Supplementary Figures 1 and 2A-F, left).

SNP analysis of the proband and her parents with an Illumina HumanHap500 Infinium 2 chip confirmed the segmental UPD-22 and positioned its break point between SNPs rs1022533 (chr22:39302493) and rs1062753 (chr22:40722757), but SNP rs10483213 (chr22:40669471) showed unexpected results (Supplementary Table 2). Sequencing analysis demonstrated that in a subset of the cells the paternal chromosome 22 carried the maternal rs10483213 allele (Table 2, Supplementary Figure 2A-F, right). This result is consistent with the occurrence of a gene conversion event. Complete sequencing of the chromosome 22 segment between 40664700 and 40678040 in all family members revealed an additional polymorphism, 22-5(G/A) at Chr22:40676675, informative for paternal UPD (Table 2). These data demonstrate that deletion and segmental UPD break points, while very close, do not coincide. The deletion break point is located between chr22:40665726 and 40665857; the segmental 
a

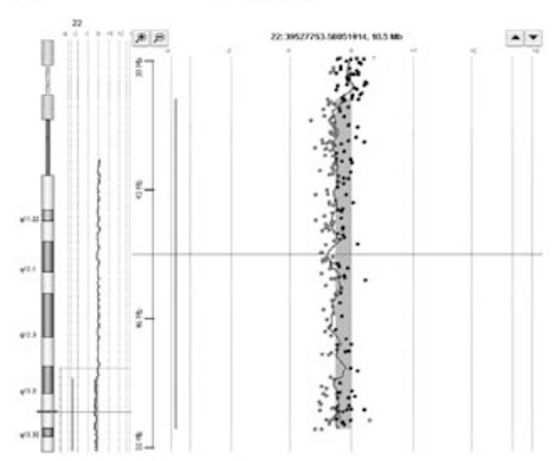

c D22S1141

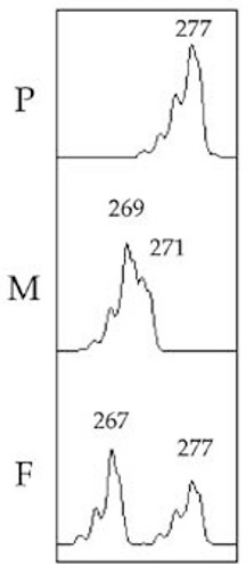

D22S1169

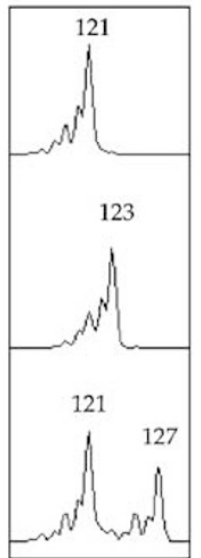

b

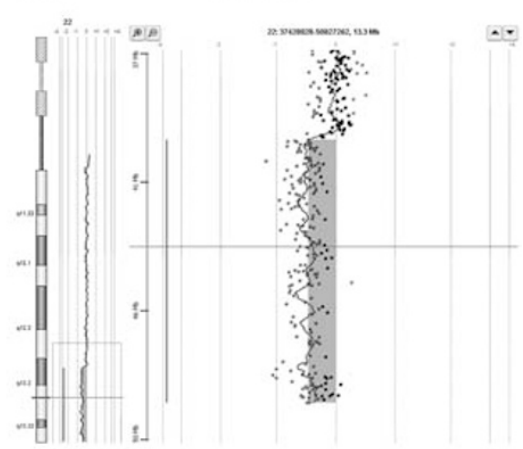

d

D22S1153

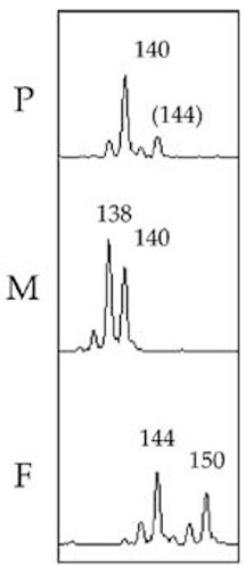

D22S1179

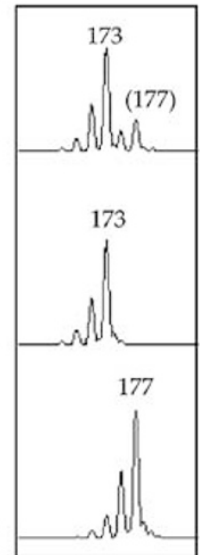

Figure 1 (a and b) (left), array CGH profile of chromosome 22 showing the deletion at 22q13.2-qter in Case 1 and Case 2, respectively. (a) (right), enlargement of Case 1 deleted region: the 22q-deletion break point lies between the oligomer at $40.659 \mathrm{Mb}$ (still present) and the oligomer at $40.666 \mathrm{Mb}$ (first deleted); probes from 40.666 to $49.468 \mathrm{Mb}$ have an average $\log _{2}$ ratio of about -0.25 , compatible with mosaic deletion; (b) (right), enlargement of Case 2 deleted region: the $22 \mathrm{q}$ deletion break point lies between the oligomer at $40.417 \mathrm{Mb}$ (still present) and the oligomer at $40.494 \mathrm{Mb}$ (first deleted); probes from 40.494 to $49.468 \mathrm{Mb}$ have an average $\log _{2}$ ratio of about -0.44 , compatible with mosaic deletion. (c and d) Parental origin of the 22q13 deletion in Cases 1 and 2, respectively. P, proband; $M$, mother; $F$, father. (c) Case 1: for both markers, the proband (P) has inherited only one paternal allele and no maternal alleles, indicating a deletion of maternal origin. Allele sizes are shown. (d) Case 2: the proband has inherited one maternal and one paternal allele in non-stoichometric proportions, indicating the presence of a mosaic deletion of paternal origin. The sizes of the mosaic deleted alleles are in commas.

UPD break point location can be narrowed down to a $7.2 \mathrm{~kb}$ region between rs10483213 and 22-5(G/A) (chr22:40669471-40676675) (Figure 2a).

To find out how the different maternal and paternal chromosomes 22 were distributed in the mosaic lines, we obtained pure EBV subclones containing either the deleted or the UPD-22 chromosome by dilution cloning. We typed all subclones for SNPs 1030-1050 and rs10483213, and for the telomere of the deleted chromosome 22 . We found that the gene conversion carrying paternal chromosome 22 is associated with the maternal 22 with segmental UPD, while the paternal chromosome 22 that did not undergo gene conversion is associated with the deleted maternal 22 (Figure $2 \mathrm{~b}$ and Supplementary Figure $2 \mathrm{G}-\mathrm{H}$ ).

In addition to blood, we also analyzed EBV lines and saliva from each proband.
We found in Case 1 the same degree of mosaicism in the proband's blood (not shown), EBV line, and saliva (Supplementary Figure 2A and I), whereas in Case 2 the level of mosaicism in different tissues seems to vary between 70 and 80\% (Supplementary Figure 3).

\section{Discussion}

Apart from patients with $\mathrm{r}(22)$ chromosomes, ${ }^{14-17}$ mosaicism for $\operatorname{del}(22)(\mathrm{q} 13)$ has so far been described in a 5.5year-old girl with global developmental delay, epilepsy, and mild facial dysmorphisms, ${ }^{18}$ and in two fetuses it was prenatally diagnosed because of ultrasound anomalies ${ }^{19}$ and increased risk of Down's syndrome determined by maternal serum screening. ${ }^{4}$ To the best of our knowledge, Case 1 represents the first patient with $22 q 13.3$ deletion 
Table 2 Molecular typing of Case 1

\begin{tabular}{|c|c|c|c|c|c|c|c|c|c|c|}
\hline \multirow[t]{2}{*}{ Locus } & \multirow[t]{2}{*}{ Position (bp) } & \multicolumn{3}{|c|}{ Blood DNA } & \multicolumn{4}{|c|}{ Somatic cell hybrid clones } & \multicolumn{2}{|c|}{$L D$ EBV clones } \\
\hline & & Proband & Mother & Father & wt 22-1 & wt $22-2$ & UPD22 & $\operatorname{del}(22)$ & $\operatorname{del}(22)$ & UPD22 \\
\hline D22S280 & 31539000 & 213 & $213 / 215$ & 213 & 213 & 213 & 213 & 213 & NT & NT \\
\hline $\mathrm{D} 22 \mathrm{~S} 1162$ & 32641000 & $157 / 151$ & $151 / 161$ & $157 / 163$ & 157 & 157 & 151 & 151 & NT & NT \\
\hline $\mathrm{D} 22 \mathrm{~S} 1158$ & 32904000 & $219 / 237$ & $237 / 219$ & $219 / 235$ & 219 & 219 & 237 & 237 & NT & NT \\
\hline 1226 G/A & 40665762 & $\mathrm{G} / \mathrm{A}$ & $\mathrm{A} / \mathrm{G}$ & G & G & G & A & Null & G & $\mathrm{G} / \mathrm{A}$ \\
\hline $1228 \mathrm{G} / \mathrm{A}$ & 40665764 & $\mathrm{~A} / \mathrm{G}$ & $\mathrm{G} / \mathrm{A}$ & A & A & A & G & Null & A & $\mathrm{A} / \mathrm{G}$ \\
\hline $1244 \mathrm{G} / \mathrm{A}$ & 40665780 & $\mathrm{G} / \mathrm{A}$ & $A / G$ & G & G & G & A & Null & G & $\mathrm{G} / \mathrm{A}$ \\
\hline 1246 G/A & 40665782 & $\mathrm{~A} / \mathrm{G}$ & $\mathrm{G} / \mathrm{A}$ & A & A & A & G & Null & A & $A / G$ \\
\hline rs10483213 & 40669471 & $A / G$ & $\mathrm{G} / \mathrm{A}$ & A & A & G & G & Null & A & G \\
\hline rs60025556 & 40673719 & A & $\mathrm{A} / \mathrm{C}$ & A & A & A & $A$ & Null & NT & NT \\
\hline rs8140869 & 40674243 & A & $\mathrm{A} / \mathrm{G}$ & A & A & A & A & Null & NT & NT \\
\hline rs5996092 & 40674354 & G & $\mathrm{G} / \mathrm{A}$ & G & G & G & G & Null & NT & NT \\
\hline $22-5 \mathrm{G} / \mathrm{A}$ & 40676675 & G & A & $G$ & $G$ & $G$ & $\mathrm{G}$ & Null & NT & NT \\
\hline D22S418 & 41748000 & 141 & $135 / 141$ & 141 & 141 & 141 & 141 & Null & NT & NT \\
\hline D22S1179 & 41922000 & 173 & $173 / 177$ & $173 / 177$ & 173 & 173 & 173 & Null & NT & NT \\
\hline D22S1141 & 44097000 & 277 & $269 / 271$ & $277 / 267$ & 277 & 277 & 277 & Null & NT & NT \\
\hline D22S1169 & 47788000 & 121 & 123 & $121 / 127$ & 121 & 121 & 121 & Null & NT & NT \\
\hline
\end{tabular}

Genomic DNAs was extracted from peripheral blood lymphocytes (blood), somatic cell hybrid clones containing isolated chromosomes 22, proband's EBV clones obtained by limiting dilution (LD). When two alleles are not in 1:1 ratio, they are printed in correspondingly larger/smaller size. Null, did not amplify; NT, not tested.

syndrome associated with a complex mosaic chromosome rearrangement consisting of a 22q13.2-qter chromosome deletion in $45 \%$ of her cells, and an apparently normal cell line that actually carries a 22q13 segmental paternal UPD in $55 \%$ of the cells.

Except for the possibility of homozygosity for recessive mutations, maternal UPD 22 does not seem to have an adverse impact on the phenotype. ${ }^{20}$ This means that no maternally imprinted gene with major effect maps to chromosome 22. The phenotypic effects of paternal UPD 22 are still unknown, as no cases have been reported. Accordingly, our proband's clinical phenotype may or may not depend entirely on the presence and level of the mosaic 22q13 deletion. Psychomotor delay was more severe in Case 2 (73\% of deleted cells in blood and over $80 \%$ in saliva) than in Case 1 (45\% deleted cells in both blood and saliva). Similarly in the case reported by Yong, ${ }^{18}$ with 95\% of deleted cells, psychomotor delay was severe (Table 1). Whatever the level of mosaicism in the brain may be, it is enough to express the full neurological phenotype of the syndrome. Haploinsufficiency of the SHANK3 gene is the main cause of the neurological phenotype in the $22 \mathrm{q} 13$ deletion syndrome. ${ }^{7-10}$ Our cases demonstrate that even in mosaicism haploinsufficiency of SHANK3 is sufficient to severely perturb its activity.

\section{Mechanisms of mosaicism}

As shown in Figure 2b, our cytogenetic and molecular analysis of Case 1 demonstrated the existence of a cell line with 22q13.2 maternal deletion and another with paternal isodisomy for the distal portion of chromosome 22q. The deleted cell line contains one normal paternal chromosome 22 and one maternal deleted chromosome 22; the
UPD line contains one maternal chromosome 22 with its terminal $8.8 \mathrm{Mb}$ derived from the paternal chromosome 22 , and a paternal chromosome 22 carrying the maternal rs10483213 allele, evidence of mitotic interaction with a maternal chromosome 22. This gene conversion is most likely associated with the recombination event leading to paternal segmental isodisomy. The paternal chromosome 22 in the line carrying a deleted maternal chromosome 22 does not show any gene conversion (Supplementary Figure 2C and D).

Segmental isodisomy is usually assumed to be produced postzygotically by a mitotic exchange between non-sister chromatids. However, both maternal and paternal UPD should than be found in daughter cells, unless the mitotic event occurs very early and the products split between embryonic and extraembryonic tissues, or one of the products is lethal, as assumed in Beckwith-Wiedemann syndrome. $^{21}$ In our case, deletion and segmental UPD break points, while very close, do not coincide: on the UPD 22 chromosome, SNPs 1226-1246 and rs10483213, all distal to the deletion break point, contain a maternal allele (Figure $2 \mathrm{a}$ and Table 2). Fish analysis also demonstrated the presence of a cell with three copies of non-deleted chromosome 22. On the basis of these findings, we hypothesize two possible mechanisms leading to the formation of this complex mosaic.

In the first model (Figure 3a), a meiotic or post-meiotic event generated a deletion on the maternally inherited chromosome 22. During replication, one deleted chromatid repaired itself through break-induced replication ${ }^{22,23}$ using one of the paternal chromatids as a template, generating a gene conversion during the process. This event resulted in the $55 \%$ Pat UPD+gene conversion 


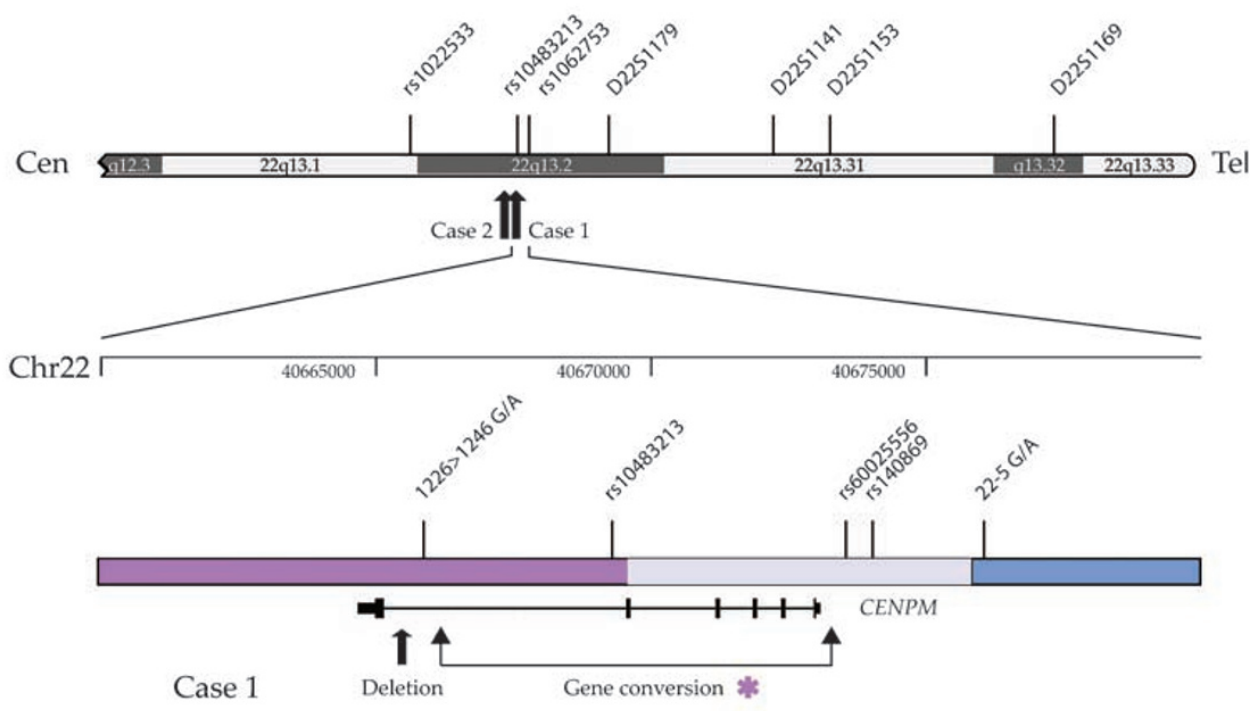

b Pat $\mathrm{UPD}(22)$

$\operatorname{Del}(22)$

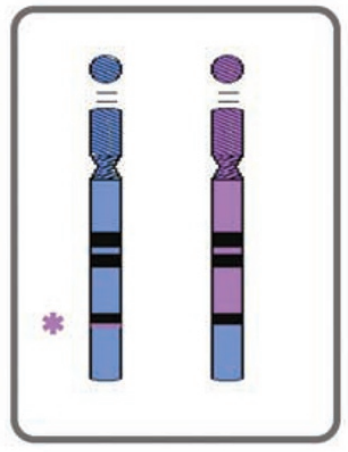

$55 \%$

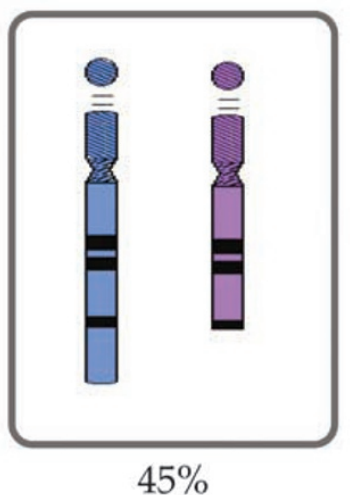

$45 \%$

Figure 2 (a) Position of the break points. The distal $15 \mathrm{Mb}$ of chromosome $22 \mathrm{q}$ are shown. The locations of cytogenetic bands and informative polymorphisms are indicated. The position of the break points in Cases 1 and 2 are marked by arrows. A blown-up detail of the $20 \mathrm{~kb}$ surrounding the Case 1 break point is also shown. Again, the locations of the CENPM gene, the deletion break point (arrow), and informative polymorphisms are indicated. The maternal portion of the chromosome is shown in pink, the paternal chromosome and segmental UPD in blue; the UPD break point region is in light blue. The upper limits of the gene conversion region are indicated by parentheses. (b) Graphic representation of the mosaic in Case 1. The two cell lines containing the deleted chromosome 22 [del(22)] or the 22q13 paternal segmental UPD [UPD(22)] are shown in gray frames. Paternal chromosome material is represented in blue, maternal material in pink. The gene conversion on one paternal chromosome 22 is indicated by a pink asterisk (*). The degree of mosaicism is also indicated.

lineage. The remaining replicated deleted maternal allele, still containing an unprotected chromosome end, lost several kilobases because of exonucleolytic activity. The secondary structure produced by the AT-rich repeat probably protected the chromosome from further degradation and facilitated stabilization by telomere healing. This is why the deletion break point of the maternal deleted chromosome 22 is located in the middle of a $1.3 \mathrm{~kb}$ AT-rich repeat.

In the second model (Figure 3b), based on FISH data showing the presence of one cell with three copies of non- deleted chromosome 22, the rearrangement occurred in a trisomic zygote with two paternal $22 \mathrm{q}$ and one maternal $22 \mathrm{q}$ chromosomes. A multiple recombination event involving all three chromosomes caused gene conversion, segmental UPD, and deletion; the deleted chromosome then lost several kilobases before being stabilized by the addition of telomeric sequences.

In Case 2, the presence of a normal cell line is, most likely, consistent with a postzygotic event, requiring a single hit to explain both the deletion and the mosaicism (Supplementary Figure 4). 

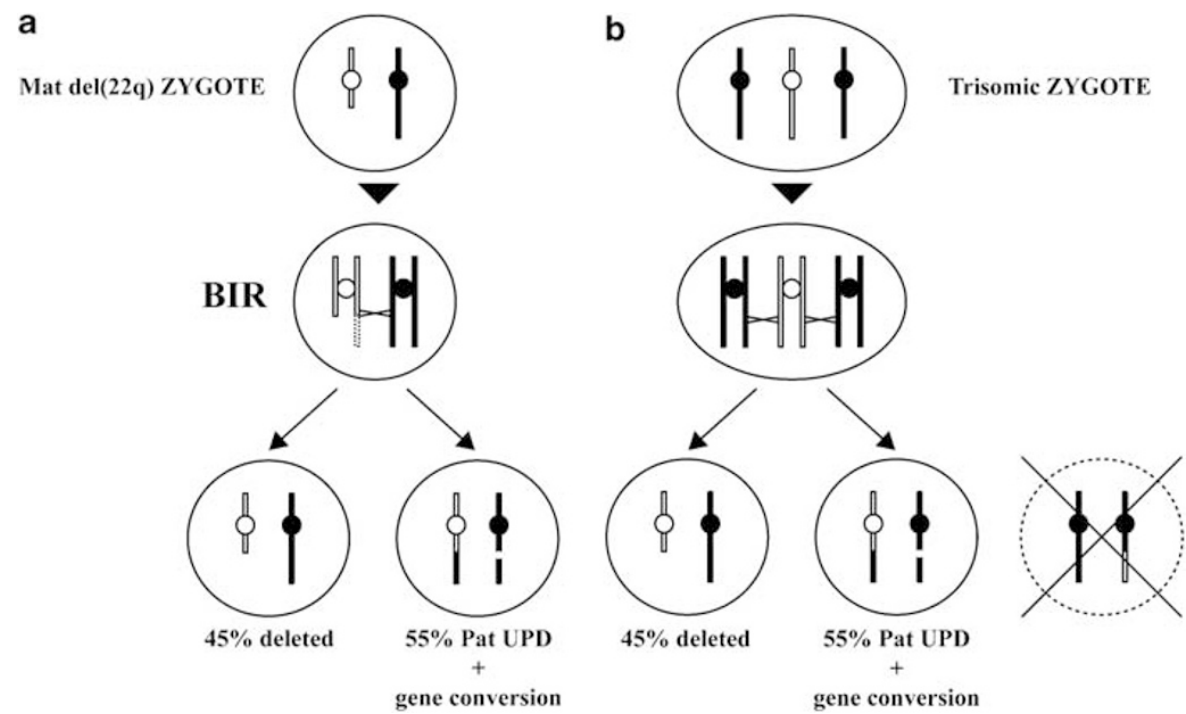

Figure 3 Mechanisms explaining the origin of the rearrangement in Case 1. (a) Rearrangement from a zygote containing a deleted maternal chromosome 22. After replication, one deleted replicated maternal allele recaptures the missing terminal portion of chromosome 22 from a paternal chromatid through BIR. During this maternal-paternal allele interaction, the paternal allele undergoes gene conversion. The products of this process form the 55\% Pat UPD + gene conversion lineage. The other replicated deleted maternal allele loses several kilobases because of exonucleolytic activity (not shown), then is stabilized by telomere healing and forms the $45 \%$ deleted lineage. (b) Postzygotic rearrangement from a trisomic zygote. Multiple recombination involving two paternal and one maternal chromosomes 22 simultaneously generates gene conversion, isodisomy, and deletion. Paternal segmental UPD and gene conversion segregate in one cell line, deletion in another. We did not observe any cells with maternal segmental UPD. Paternal chromosome 22 is represented by a black-filled bar, maternal 22 by an empty bar.

A break during DNA replication in the zygote would generate a deleted chromatid, immediately stabilized by telomere healing. Segregation gave rise to a cell line with normal karyotype and another with 22q13 deletion.

In conclusion, we have described two patients with mosaic (45-75\%) terminal 22q13 deletions, one of whom additionally carries a mosaic 22q13 segmental UPD (Case 1).

Keeping in mind that both cases showed the same 22q13 deletion size:

(1) the clinical phenotype, much more severe in case 2 than in case 1 , should depend entirely on the presence of the mosaic 22q13 deletion; mosaic segmental UPD22 probably does not have relevant clinical impact.

(2) the complex mosaicism in Case 1 originated from a postzygotic or early embryonic recombination event causing a gene conversion event leading to the formation of segmental UPD and terminal deletion, both retained in mosaic in the fetus. Mosaicism in Case 2 was also probably due to a postzygotic event.

\section{Acknowledgements}

We thank the anonymous reviewers for their suggestions. This study was supported by Telethon Grant GGP06208A to MCB and RG; by
PRIN 2005 and 2006 (to OZ and ER), Fondazione Mariani and Fondazione CARIPLO (all to OZ). Part of this study was supported by the Wellcome Trust.

\section{References}

1 Starke H, Mitulla B, Beensen V et al: First postnatal case of mosaic del(22)/r(22). Prenat Diagn 2003; 23: $765-767$.

2 Frizzley JK, Stephan MJ, Lamb AN et al: Ring 22 duplication/ deletion mosaicism: clinical, cytogenetic, and molecular characterisation. J Med Genet 1999; 36: 237-241.

3 Bergman A, Blennow E: Inv dup(22), del(22)(q11) and r(22) in the father of a child with DiGeorge syndrome. Eur J Hum Genet 2000; 8: 801-804.

4 Phelan MC, Brown EF, Rogers RC: Prenatal diagnosis of mosaicism for deletion 22q13.3. Prenat Diagn 2001; 21: 1100.

5 Lindquist SG, Kirchhoff M, Lundsteen C et al: Further delineation of the 22q13 deletion syndrome. Clin Dysmorphol 2005; 14: $55-60$.

6 Phelan MC, Rogers RC, Saul RA et al: 22q13 deletion syndrome. Am J Med Genet 2001; 101: 91-99.

7 Bonaglia MC, Giorda R, Borgatti R et al: Disruption of the ProSAP2 gene in a $\mathrm{t}(12 ; 22)(\mathrm{q} 24.1 ; \mathrm{q} 13.3)$ is associated with the $22 \mathrm{q} 13.3$ deletion syndrome. Am J Hum Genet 2001; 69: 261-268.

8 Wilson HL, Wong AC, Shaw SR et al: Molecular characterisation of the 22q13 deletion syndrome supports the role of haploinsufficiency of SHANK3/PROSAP2 in the major neurological symptoms. J Med Genet 2003; 40: 575-584.

9 Durand CM, Betancur C, Boeckers TM et al: Mutations in the gene encoding the synaptic scaffolding protein SHANK3 are associated with autism spectrum disorders. Nat Genet 2007; 39: 25-27.

10 Moessner R, Marshall CR, Sutcliffe JS et al: Contribution of SHANK3 mutations to autism spectrum disorder. Am J Hum Genet 2007; 81: 1289-1297. 
11 Bonaglia MC, Giorda R, Mani E et al: Identification of a recurrent breakpoint within the SHANK3 gene in the 22q13.3 deletion syndrome. J Med Genet 2006; 43: 822-828.

12 Bonaglia MC, Marelli S, Gottardi G et al: Subtelomeric trisomy 21q: a new benign chromosomal variant. Eur J Med Genet 2007; 50: $54-59$.

13 Giorda R, Cerritello A, Bonaglia MC et al: Selective disruption of muscle and brain-specific BPAG1 isoforms in a girl with a $6 ; 15$ translocation, cognitive and motor delay, and tracheo-oesophageal atresia. J Med Genet 2004; 41: e71.

14 Stoll C, Roth MP: Segregation of a 22 ring chromosome in three generations. Hum Genet 1983; 63: 294-296.

15 Tommerup N, Warburg M, Gieselmann V, Hansen BR, Koch J, Petersen GB: Ring chromosome 22 and neurofibromatosis. Clin Genet 1992; 42: 171-177.

16 Woods CG, Bankier A, Curry J et al: Asymmetry and skin pigmentary anomalies in chromosome mosaicism. J Med Genet 1994; 31: 694-701.

17 Chen CP, Chern SR, Chang TY et al: Prenatal diagnosis of mosaic ring chromosome 22 associated with cardiovascular abnormal- ities and intrauterine growth restriction. Prenat Diagn 2003; 23: $40-43$.

18 Yong YP, Knight LA, Yong MH, Lam S, Ho LY: Partial monosomy for chromosome 22 in a girl with mental retardation. Singapore Med J 1997; 38: 85-86.

19 Riegel M, Baumer A, Wisser J, Acherman J, Schinzel A: Prenatal diagnosis of mosaicism for a $\operatorname{del}(22)(\mathrm{q} 13)$. Prenat Diagn 2000; 20: $76-79$.

20 Schinzel AA, Basaran S, Bernasconi F, Karaman B, Yuksel-Apak M, Robinson WP: Maternal uniparental disomy 22 has no impact on the phenotype. Am J Hum Genet 1994; 54: 21-24.

21 Henry I, Puech A, Riesewijk A et al: Somatic mosaicism for partial paternal isodisomy in Wiedemann-Beckwith syndrome: a postfertilization event. Eur J Hum Genet 1993; 1: 19-29.

22 Meselson M, Weigle JJ: Chromosome breakage accompanying genetic recombination in bacteriophage. Proc Natl Acad Sci USA 1961; 47: 857-868.

23 Kraus E, Leung WY, Haber JE: Break-induced replication: a review and an example in budding yeast. Proc Natl Acad Sci USA 2001; 98: $8255-8262$.

Supplementary Information accompanies the paper on European Journal of Human Genetics website (http://www.nature.com/ejhg) 